\title{
Select All That Apply
}

National Cancer Institute

\section{Source}

National Cancer Institute. Select All That Apply. NCI Thesaurus. Code C157108.

A directive to choose all that apply to the situation. 